P64 (continued)

tap) to make infant formula, and young children's bottled water intake. Mothers with friends or family in Flint during the Flint Water Crisis also drank more bottled water and gave their children bottled water more often than mothers without. Mothers' negative perceptions of tap water in general were associated with greater sugar-sweetened beverage intake among mothers and young children. On average, mothers spent $\$ 27 /$ month on bottled water.

Conclusion: Distrust of tap water is common among lowincome Michigan mothers. This distrust creates an economic and health burden due to reliance on bottled water and may increase socioeconomic inequalities in nutrition. Community-based interventions that improve trust and increase knowledge of tap water safety among low-income women are needed.

Funding: Robert Wood Johnson Foundation, NIH.

\section{P65 The Role of Older Adults in 2- and 3- Generation Households: Implications for Diet Quality and Household Food Security}

Joshua Brown, BS, School of Health \& Rehabilitation Sciences, College of Medicine, The Ohio State University; Patrick Creedon, MPA, School of Health \& Rehabilitation Sciences, College of Medicine, The Ohio State University; Christopher Taylor, PhD, LD, RDN, FAND, School of Health $\&$ Rehabilitation Sciences, College of Medicine, The Ohio State University; Kammi Schmeer, PhD, Department of Sociology, College of Arts \& Sciences, The Ohio State University; Neal Hooker, PhD, John Glenn College of Public Affairs, The Ohio State University; Jill Clark, PhD, John Glenn College of Public Affairs, The Ohio State University; Jennifer Garner, PhD, RD, jennifer.garner@osumc.edu, School of Health \& Rehabilitation Sciences, College of Medicine, The Ohio State University, 453 West 10th Ave, Columbus, $\mathrm{OH}, 43210$

Background: Food insecurity rates have declined overall (pre-COVID), but have increased significantly among older adults in the past decade. The majority of older adults in the U.S. also have suboptimal diet quality. An older adult's household role may influence their diet quality and the household's food security status, especially in mixed-generation households, but this hypothesis has yet to be tested.

Objective: To explore the relationships between an older adult's household role, the household's food security status, and the older adult's diet quality in mixed-generation households.

Study Design, Settings, Participants: A cross-sectional analysis of a nationally-representative sample of U.S. households with at least 1 older adult (age 60+ years) from the 2011-2016 NHANES was conducted $(n=8,136)$. Households were categorized as: older adult as head of household and child caregiver (ie, child(ren) present, but no adults 18-59 years; HHC); head of household, but not a caregiver (HHNC); and neither head of household nor caregiver (ie, reference person $<60$ years; NHNC). The U.S. Household Food Security Survey and Healthy Eating
Index-2015 (HEI-2015) from a single dietary recall were used to assess food security status and diet quality, respectively.

Measurable Outcome/Analysis: Weighted chi-square and ANOVA tests were used to compare food security status and HEI-2015 scores across household categories, respectively.

Results: HHNC households were significantly more likely to be fully food secure $(84 \%$ FFS; $P<0.001)$ than HHC households (67\% FFS) and NHNC households (68\% FFS). Older adults as HHNC had significantly higher HEI-2015 scores (mean: 53.7, $P<0.001$ ) than HHC older adults (50.2) and NHNC older adults (51.5).

Conclusion: Findings suggest that caregiving demands may have a negative influence on food security and diet quality of older adults in mixed generation households. While such relationships have potential bearing on nutrition programming and policy, further research is needed to understand the underlying household dynamics, including more advanced analyses to account for potential covariates (eg, the number and age of children and roles of other household members).

Funding: The Ohio State University Institute for Population Research via a center grant (P2CHD058484) awarded by the National Institute of Child Health and Human Development.

\section{P66 Understanding How the COVID-19 Pandemic Influenced Older Adults' Grocery Shopping Habits}

Frankie Palmer, BS, fapalmer@crimson.ua.edu, The University of Alabama, 486 Russell Hall Box 870311, Tuscaloosa, AL, 35487; Seung Eun Jung, PhD, RDN, The University of Alabama; Mackinsey K. Shahan, MS, RDN, The University of Alabama; Amy Ellis, PhD, MPH, LD, RDN, The University of Alabama

Background: The ability to grocery shop is an important aspect of maintaining adequate nutritional status among older adults. During the COVID-19 pandemic, many older adults changed their typical grocery shopping habits attempting to remain safe.

Objective: To understand how the COVID-19 pandemic influenced older adults' grocery shopping habits.

Study Design, Setting, Participants: In this qualitative study, a total of 23 older adults aged 60 years or older participated in individual interviews via Zoom.

Measurable Outcome/Analysis: Data were analyzed using directed content analysis.

Results: Attempting to be cautious, most participants reported altering grocery shopping habits by shopping less frequently, using online services such as grocery pickup or delivery, or having friends or family members do the shopping. However, some participants continued shopping in stores due to poor quality produce items picked out by shoppers and safe policies implemented by stores. Also, grocery shopping in stores became incentivized as some participants viewed it as a form of entertainment, social interaction, and even physical activity during the pandemic. Although many Continued on page S55 